\title{
COVID-19 and the nervous system
}

\author{
Joseph R. Berger ${ }^{1,2}$
}

Received: 31 March 2020 / Revised: 31 March 2020 / Accepted: 2 April 2020/Published online: 23 May 2020

(C) Journal of NeuroVirology, Inc. 2020

\begin{abstract}
A pandemic due to novel coronavirus arose in mid-December 2019 in Wuhan, China, and in 3 months' time swept the world. The disease has been referred to as COVID-19, and the causative agent has been labelled SARS-CoV-2 due to its genetic similarities to the virus (SARS-CoV-1) responsible for the severe acute respiratory syndrome (SARS) epidemic nearly 20 years earlier. The spike proteins of both viruses dictate tissue tropism using the angiotensin-converting enzyme type 2 (ACE-2) receptor to bind to cells. The ACE-2 receptor can be found in nervous system tissue and endothelial cells among the tissues of many other organs.

Neurological complications have been observed with COVID-19. Myalgia and headache are relatively common, but serious neurological disease appears to be rare. No part of the neuraxis is spared. The neurological disorders occurring with COVID-19 may have many pathophysiological underpinnings. Some appear to be the consequence of direct viral invasion of the nervous system tissue, others arise as a postviral autoimmune process, and still others are the result of metabolic and systemic complications due to the associated critical illness. This review addresses the preliminary observations regarding the neurological disorders reported with COVID-19 to date and describes some of the disorders that are anticipated from prior experience with similar coronaviruses.
\end{abstract}

Keywords COVID-19 $\cdot$ Coronavirus $\cdot \mathrm{SARS}-\mathrm{CoV}-2 \cdot$ Neurological complications $\cdot$ Meningitis $\cdot$ Stroke $\cdot$ Encephalitis $\cdot$ Myositis

\section{Background}

In mid-December 2019, patients with an unusual pneumonia were recognized in Wuhan, China. On December 31, 2019, this new infection was reported to the World Health Organization 31, 2019 (CNN 2020). By January 7, 2020, genetic analysis of viral isolates from affected patients indicated that the etiologic agent was a novel coronavirus distinct from those causing severe acute respiratory syndrome (SARS) and middle east respiratory syndrome (MERS). By January 16, 2020, it is recognized in Japan in a Japanese man who had travelled to Wuhan (CNN 2020). From the time of its appearance in December 2019, this novel coronavirus spread a lightening-like speed through the world's population. As of March 29, 2020, the illness referred to as coronavirus disease

Joseph R. Berger

joseph.berger@pennmedicine.upenn.edu

1 Department of Neurology, Perelman School of Medicine, University of Pennsylvania, Philadelphia, PA, USA

2 Department of Neurology, Perelman School of Medicine, Perelman Center for Advanced Medicine, Room 765 South Tower, Philadelphia, PA 19104, USA
2019 (COVID-19) has been recognized in 172 of the world's 192 countries (Organization WH 2020). The total number of confirmed cases was 634,835 , and the number of deaths attributed to the disease was 29,957 (Organization WH 2020) and rapidly rising.

\section{The coronavirus:SARS-CoV-2}

Coronaviruses, the family of viruses to which the virus causing COVID-19 belongs, were first identified in humans in 1965 from a child with an upper respiratory infection (McIntosh 1985). Serologic surveys have indicated a worldwide distribution, and depending on the season, they are responsible for 1 to $35 \%$ of upper respiratory infections (McIntosh 1985). Community-acquired coronavirus was chiefly associated with a mild upper respiratory infection. However, the severe acute respiratory syndrome (SARS) in 2002-2003 which affected more than 8000 persons worldwide with a nearly $10 \%$ mortality (CDC 2020) and the middle east respiratory syndrome (MERS) epidemic that started in 2012 affected nearly 2500 persons with a nearly $35 \%$ mortality (Organization, W.H 2020) were caused by coronaviruses. 
Coronaviruses are enveloped viruses measuring 100$150 \mathrm{~nm}$ in diameter with a positive-sense single-stranded RNA genome (McIntosh 1985). The nucleocapsid demonstrates a helical symmetry. By electron microscopy, coronaviruses display spike peptomer projections measuring $20 \mathrm{~nm}$ in length that are responsible for the virus' tropism. It is the "crown-like" appearance of these projections that gave rise to the name coronavirus. Coronaviruses have four main structural proteins: spike (S)-protein, membrane (M), envelope (E), and nucleocapsid $(\mathrm{N})$-proteins that are all encoded with in the $3^{\prime}$ end of the virus (Fehr and Perlman 2015). The spike protein is a trimeric fusion protein that mediates attachment to the host receptor. The membrane protein is the most abundant structural protein in the virion. The envelope protein facilitates assembly and release of the virus, and the ion channel activity in SARS-CoV envelope protein is required for pathogenesis. The N-protein constitutes the only protein present in the nucleocapsid. It is composed of 2 domains that bind RNA (Fehr and Perlman 2015). The hemagglutinin-esterase protein is present in a subset of $\beta$-coronaviruses and binds sialic acids on surface glycoproteins and contains acetylesterase activity (Li 2016).

The attachment of the virus to the host cell is initiated by the S-protein and its receptor. This interaction is the primary determinant for tissue tropism (Fehr and Perlman 2015). Different coronaviruses have an affinity for different receptors. As stated, SARS-CoV-2 interacts with the ACE-2 receptor (Fehr and Perlman 2015).

Coronaviruses are classified into four genera: alpha, beta, gamma, and delta. Coronaviruses in the beta genus include SARS, MERS, and the new coronavirus, referred to as SARS-Cov-2. There are now at least six human coronaviruses including SARS-CoV-1, SARSCoV-2, MERS-CoV, HcoV-OC43, HCoV-229E, HCoVNL-63, and HCoV-HKU1 (Arabi et al. 2015). SARS$\mathrm{CoV}-2$ was named for its close genetic relationship to the SARS virus. The "spike proteins" of both SARS (Imai et al. 2010) and SARS-CoV-2 (Lu et al. 2020) use the angiotensin-converting enzyme receptor type 2 (ACE-2) to bind to cells. The ACE-2 receptor is widely distributed in the body present in the lungs, oral and nasal mucosa, bone marrow and spleen, skin, heart, arteries, kidneys, adipose tissue, reproductive system, and brain (Hamming et al. 2004). ACE 2 receptor is also expressed in the central nervous system predominantly in thalamic nuclei, cerebellum, and inferior olivary nuclei (Allen et al. 2009). The location of these receptors in the brain may be of relevance with respect to coronavirus encephalitis. The SARS-CoV-2 genome is 29,903 bases long, and estimates of viral evolution suggest that it arose no earlier than the end of October
2019. A $96 \%$ homology with bat coronavirus indicates that it originated as a bat virus with an intermediate host in another animal (Chan et al. 2020). Two different strains of SARS-CoV-2 have been identified: an S-strain which is evolutionary older and less aggressive and an L-strain which is more aggressive and spreads more quickly (Tang et al. 2020). The L-strain is more prevalent $(70 \%)$ than the S-strain (30\%) (Tang et al. 2020).

\section{General clinical manifestations of COVID-19}

Coronaviruses in the animal kingdom have been associated with a variety of disorders, including infectious bronchitis and nephrosis in chickens; gastroenteritis and encephalitis in young piglets; enteritis in turkey, dogs, and calves; hepatitis and encephalitis in mice; pneumonitis and sialodacryoadenitis in rats; and infectious peritonitis in cats. Coronaviruses are a widely distributed and frequent cause of upper respiratory infections (McIntosh and Anderson 2005). The overwhelming identified coronavirus infections in man have been respiratory infections, chiefly mild upper respiratory infections. However, gastrointestinal disease has been observed in neonates and infants (McIntosh and Anderson 2005).

In a study from the Chinese Centers for Disease Control, $80.9 \%$ of persons infected with SARS-CoV-2 were asymptomatic or developed only mild pneumonia yet shed large amounts of virus in the early phase of their infection (Wang et al. 2020a). In those becoming symptomatic, the clinical manifestations of COVID-19 are virtually indistinguishable from influenza. It is initially characterized by fever in up to $90 \%$ and cough in approximately 50\% (Guan et al. 2020; Wang et al. 2020b; Tian et al. 2020) with symptoms developing after a median incubation period of 4-6.7 days (Tian et al. 2020; Xu et al. 2020a). Myalgia and fatigue are seen in about $50 \%$ (Huang et al. 2020). Anosmia has been a common early symptom (Hopkins and Kumar 2020). Headache has been reported in less than $10 \%$ (Tian et al. 2020). Acute myocarditis has been observed in as many as $12 \%$ (Huang et al. 2020), and gastroenteritis and diarrhea are seen in about 5\% (Guan et al. 2020). On chest CT scan, the most common abnormality was ground glass opacities seen in about 75\% (Pan et al. 2020). Most patients are diagnosed with pneumonia. Older patients have more severe disease. In one study from China, mechanical ventilation was required in $6.1 \%$ of patients (Guan et al. 2020), though rates of acute respiratory distress syndrome as high as $29 \%$ have been reported. A study of 262 confirmed cases revealed that $17.6 \%$ had severe disease, whereas $73.3 \%$ were assessed as mild, $4.2 \%$ non-pneumonic, and $5.0 \%$ asymptomatic (Tian et al. 2020), though it is believed that widespread testing would reveal far higher numbers of asymptomatic or mildly symptomatic persons. Lymphopenia is 
commonly observed (Huang et al. 2020; Wang et al. 2020c), and severe lymphopenia may be associated with a higher mortality (Tan et al. 2020). Acute phase reactants are elevated including a variety of cytokines, such as TNF $\alpha$, IL6, and IL10 (Wang et al. 2020b; Huang et al. 2020). Case fatalities rates have varied by country with the highest reported in Bangladesh at $11.36 \%$ and the lowest in Israel at $0.30 \%$ (Oke and Heneghan 2020). Mortality is higher with advanced age and underlying comorbidities (Wang et al. 2020c; Yang et al. 2020) including cardiac and respiratory disorders and immunosuppressed states. Children typically have milder disease, and death in children has been rare (Ludvigsson 2020).

\section{Neurological complications of COVID-19}

Severe neurological complications of COVID-19 appear to be both infrequent and diverse in nature. Virtually any part of the neuraxis appears to be susceptible to injury with SARS-CoV2. Neurological disease may be the consequence of generalized cardiorespiratory failure and metabolic abnormalities triggered by the infection, direct invasion of the virus, or an autoimmune response to the virus. Only the latter two will be discussed.

In an, as yet, unpublished report from Wuhan, China, 78 (36.4\%) of 214 COVID-19 hospitalized patients had some form of neurological disorder (Mao et al. 2020). The most common complaints were dizziness, headache, hypogeusia, and hyposmia (Mao et al. 2020). Neurological disorders were more significantly common in the severely ill and included stroke in $6(2.8 \%)$, impaired levels of consciousness in 16 (7.5\%), and muscle injury in 23 (10.7\%) (Mao et al. 2020).

The SARS epidemic of 2002-2003 may provide some reference for the frequency and nature with which neurological disorders may be seen with COVID-19. In a study of 664 suspected SARS patients in Taiwan, three patients developed an axonal polyneuropathy 3-4 weeks after disease onset (Tsai et al. 2005). Two patients developed myopathy and three rhabdomyolysis which were attributed to critical illness neuropathy and myopathy, though viral invasion could not be fully excluded (Tsai et al. 2005). Five developed large vessel ischemic stroke believed to be the consequence of multiple factors (Tsai et al. 2005). Therefore, fewer than $2 \%$ of the patients with SARS in this series were recognized as having a neurological complication. Laboratory studies in mice that are transgenic for ACE-2 receptor have demonstrated that SARS-CoV will enter the brain through the olfactory nerve and result in brain infection, but there is little cellular infiltration (Netland et al. 2008). Death in these infected transgenic mice has been attributed to respiratory failure from medullary infection. Some investigators have suggested that a similar mechanism of respiratory may attend infection with SARS-CoV-2 ( $\mathrm{Li}$ et al. 2020a).

\section{Direct effects of SARS-CoV-2 on the nervous system}

\section{Headache}

Headache is a common symptom of COVID-19 being observed in up to $40 \%$ of patients (Ding et al. 2020). Typically, the headache has been regarded as mild. The frequency with which headache occurs due to a viral meningitis remains uncertain and will require further study. Similarly, the contribution of CNS viral invasion by SARS-CoV-2 contributes to the frequently observed altered levels of consciousness in the severely ill COVID-19 patient as opposed to general systemic disease. There is a least one report of the detection of SARSCoV-2 from the CSF of a symptomatic man (Michael and Easton 2020).

\section{Meningitis/encephalitis}

Central nervous system disorders have been reported with other coronaviruses; therefore, they should not be unanticipated with SARS-CoV-2. SARS-CoV-1 has been detected by polymerase chain reaction in the cerebrospinal fluid of a 32year-old woman with SARS presenting with generalized tonic-clonic seizures (Lau et al. 2004) and in a 59-year-old woman with IgA nephropathy and SARS presenting with encephalopathy and intractable seizures (Hung et al. 2003). In a study of children hospitalized with respiratory disease and an acute encephalitis-like syndrome, approximately $12 \%$ had evidence of an acute coronavirus infection (Li et al. 2016). There are reports of an encephalitis (Arabi et al. 2015) including Bickerstaff's encephalitis (Kim et al. 2017) complicating MERS. Magnetic resonance imaging studies showed hyperintense signal abnormalities on T2-weighted imaging within the deep and subcortical hemispheric white matter, the corpus callosum, and the basal ganglia (Arabi et al. 2015).

The case reports of encephalitis with COVID-19 are limited, but an acute necrotizing encephalopathy has been reported in a woman in her 50s presenting with cough, fever, and altered mental status (Poyuiadji et al. 2020). Brain MRI showed hemorrhagic rim enhancing lesions within both thalami, medial temporal lobes and subinsular regions (Poyuiadji et al. 2020).

Unsurprisingly, encephalopathy has been described. For instance, Florida physicians reported a 74-year-old-man with multiple underlying comorbidities including atrial fibrillation, cardioembolic stroke, and Parkinsons disease, developed headache and severe encephalopathy in association with pneumonia (Filatov et al. 2020). A CT scan revealed an area of encephalomalacia in the left temporal lobe. An EEG showed diffuse and focal slowing with sharply contoured waves over the left temporal lobe. CSF showed 4 white cells with a slight elevation of protein at $68 \mathrm{mg} / \mathrm{dL}$ and normal glucose (Filatov et al. 2020). Patients with encephalopathy 
consequent to overwhelming infection and associated co-morbidities are likely to be the most common neurological complication.

\section{Stroke}

Several pathophysiological processes may be responsible for an increased risk of stroke with COVID-19. Severe disease is seen more often in older patients who often have comorbidities that increase their risk of stroke. As with SARS (Tsai et al. 2005), a hypercoagulable syndrome may complicate COVID19. Markers of coagulation may be increased during infection (Zhou et al. 2020), and disseminated intravascular coagulation has been observed (Tang et al. 2020). A viral myocarditis (Madjid et al. 2020) may increase the risk of stroke. Lastly, brain endothelial express ACE- 2 receptor and the potential exists for viral-induced vasculitis. Risk may be increased for both arterial and venous cerebrovascular disease. In one recent series of 78 COVID-19 patients, stroke occurred in $6(2.8 \%)$ (5 ischemic and 1 hemorrhagic) (Mao et al. 2020). In a small series of 24 patients from the Seattle region, stroke was observed in $2(8 \%)$ of 24 hospitalized COVID-19 patients (Bhatraju et al. 2020).

\section{Anosmia and ageusia}

Loss of smell in association with upper respiratory infection is common and has been attributed to damage to olfactory epithelial by the causative virus (Seiden 2004). Some investigators have proposed that the frequency with which URI-related olfactory loss is seen correlates with the type of virus responsible for the infection (Seiden 2004). Anosmia has been reported in up to 30 to $66 \%$ and may be an early symptom of infection (Hopkins and Kumar 2020). Anecdotally, in mild infection, it may be the only clue of infection. Anosmia is often accompanied by ageusia (Guan et al. 2020; Zhou et al. 2020). Anosmia and ageusia related to COVID-19 typically resolve over several weeks. Anosmia did not appear to be a commonly reported manifestation during the SARS epidemic as there is but one published case report (Hwang 2006). A high expression of ACE-2 receptor on oral mucosa has been reported (Xu et al. 2020b) and may be related to these symptoms with SARS-CoV-2.

\section{Myalgia/myositis}

Muscle pain is one of the most common manifestations reported in 35 to $50 \%$ of patients (Xu et al. 2020a; Huang et al. 2020; Ding et al. 2020; Li et al. 2020b). Rhabdomyolysis may occur as a late complication and can result in lifethreatening renal impairment (Jin and Tong 2020). Careful monitoring of renal and muscle enzymes is important during SARS-CoV-2 infection.

\section{Neurological autoimmune disorders with COVID-19}

Many viral illnesses may trigger an autoimmune response affecting the central or peripheral nervous system. Both acute disseminated encephalomyelitis (ADEM) and acute inflammatory demyelinating peripheral neuropathy (AIDP) may be consequences of the infection.

\section{AIDP/Guillain-Barre syndrome}

A 5-year-old child presenting with lower extremity pain, difficulty walking, peripheral facial weakness, and bulbar palsy occurring in association with $\mathrm{HCoV}-\mathrm{OC} 43$ infection has been reported (Sharma et al. 2019). MERS has also triggered a post-infectious brainstem encephalitis and Guillain-Barre syndrome (Kim et al. 2017). Reports of transverse myelitis with SARS-CoV2 are also beginning to emerge (Wang et al. 2020b).

\section{Acute disseminated encephalomyelitis (ADEM)}

As many as $75 \%$ of patients with ADEM have had a preceding viral infection or vaccination. The available literature suggests that ADEM is a rare complication of human coronavirus infection. ADEM has been described with the human coronavirus OC43 infection in a 15-year-old boy with the history of a URI the week before the development of the heralding manifestation of lower extremity numbness (Yeh et al. 2004). HCoV-OC43 was detected by PCR in CSF and nasopharyngeal secretions as well as rising antibody titers to the virus (Yeh et al. 2004). To date, there are no classic cases of ADEM described in association with SARS-CoV-2; however, a 66-year-old critically ill man developed an acute thoracic myelitis at the height of illness with SARS-CoV-2 infection (Zhao et al. 2020). Although described as post-infectious in nature, the possibility of an acute myelitis secondary to viral invasion was considered by the authors who discuss the expression of the ACE-2 receptor on spinal cord neuron membranes (Zhao et al. 2020).

\section{Discussion}

Although only 3 months have elapsed since the onset of the COVID-19 pandemic, neurological disorders do not appear to be extraordinarily common. Headache is common. Whether the headache is the consequence of viral invasion remains uncertain. Myalgias are also common and may be accompanied by an increase in muscle enzymes suggestive of a myositis, but severe rhabdomyolysis remains rare. Stroke has occurred in a small percentage of patients and likely results from multiple underlying pathophysiologic mechanisms. Although encephalopathy is seen with severe illness, it may be 
explained by the accompanying metabolic abnormalities attending a cytokine storm rather than occurring from viral invasion of the brain, though rare cases of the latter seem to occur. As with other viral infections, a small number of patients are likely to experience autoimmune neurological disease, specifically AIDP and ADEM, in the wake of SARS$\mathrm{CoV}-2$ infection.

No treatment has demonstrated high level evidence of proven efficacy, yet more than 200 clinical trials are underway around the world. Among the proposed treatments are the antimalarials (chloroquine and hydroxychloroquine), HIV protease inhibitor drugs, remdesivir, the anti-IL6 monoclonal antibody tocilizumab, and convalescent serum or immunoglobulin. To quote Shakespeare, "Diseases desperate grown, By desperate measure are relieved, Or not at all." Aggressive supportive care may require prolonged intubation. The median hospital stay for those discharged alive is 10 days (Wang et al. 2020c).

\section{References}

Allen AM et al (2009) Neuronal angiotensin. In: Squire LR (ed) Encyclopedia of Neuroscience. Academic Press, New York, pp 697-702

Arabi YM, Harthi A, Hussein J, Bouchama A, Johani S, Hajeer AH, Saeed BT, Wahbi A, Saedy A, AlDabbagh T, Okaili R, Sadat M, Balkhy H (2015) Severe neurologic syndrome associated with middle east respiratory syndrome corona virus (MERS-CoV). Infection 43(4):495-501

Bhatraju PK et al (2020 [cited 2020 March 31]; Available from:) Covid19 in critically ill patients in the Seattel region - case series. N Engl J Med. https://doi.org/10.1056/NEJMoa2004500

CDC (2020) SARS basic fact sheet. Severe Acute Respiratory Syndrome (SARS) [cited 2020 March 29]; Available from: https://www.cdc. gov/sars/about/fs-sars.html

Chan JF, Kok KH, Zhu Z, Chu H, To KK, Yuan S, Yuen KY (2020) Genomic characterization of the 2019 novgersel human-pathogenic coronavirus isolated from a patient with atypical pneumonia after visiting Wuhan. Emerg Microbes Infect 9(1):221-236

CNN (2020) Coronavirus outbreak timeline fast facts. CNN Health [cited 2020 March 29]; Available from: https://www.cnn.com/2020/02/ 06/health/wuhan-coronavirus-timeline-fast-facts/index.html

Ding Q et al (2020) The clinical characteristics of pneumonia patients coinfected with 2019 novel coronavirus and influenza virus in Wuhan, China. J Med Virol

Fehr AR, Perlman S (2015) Coronaviruses: an overview of their replication and pathogenesis. Methods Mol Biol 1282:1-23

Filatov A, Sharma P, Hindi F, Espinosa PS (2020) Neurological complications of coronavirus disease (COVID-19): encephalopathy. Retrieved April 2, 2020, from https://www.cureus.com/articles/ 29414-neurological-complications-of-coronavirus-disease-covid19-encephalopathy

Guan WJ, Ni ZY, Hu Y, Liang WH, Ou CQ, He JX, et al. (2020) Clinical Characteristics of Coronavirus Disease 2019 in China. N Engl J Med https://www.ncbi.nlm.nih.gov/pubmed/32109013

Hamming I, Timens W, Bulthuis ML, Lely AT, Navis G, van Goor H (2004) Tissue distribution of ACE2 protein, the functional receptor for SARS coronavirus. A first step in understanding SARS pathogenesis. J Pathol 203(2):631-637

Hopkins C, Kumar N (2020) Loss of sense of smell as a marker of COVID-19 infection. ENTUK [cited 2020 March 30]; Available from: https://www.entuk.org/sites/default/files/files/Loss $\% 20$ of $\%$ 20sense $\% 20$ of $\% 20$ smell $\% 20$ as $\% 20$ marker\%20of\%20COVID.pdf

Huang C, Wang Y, Li X, Ren L, Zhao J, Hu Y, Zhang L, Fan G, Xu J, Gu X, Cheng Z, Yu T, Xia J, Wei Y, Wu W, Xie X, Yin W, Li H, Liu M, Xiao Y, Gao H, Guo L, Xie J, Wang G, Jiang R, Gao Z, Jin Q, Wang J, Cao B (2020) Clinical features of patients infected with 2019 novel coronavirus in Wuhan, China. Lancet 395(10223):497-506

Hung EC et al (2003) Detection of SARS coronavirus RNA in the cerebrospinal fluid of a patient with severe acute respiratory syndrome. Clin Chem 49(12):2108-2109

Hwang CS (2006) Olfactory neuropathy in severe acute respiratory syndrome: report of A case. Acta Neurol Taiwan. 15(1):26-28. https:// www.ncbi.nlm.nih.gov/pubmed/32171076

Imai $Y$ et al (2010) Angiotensin-converting enzyme 2 (ACE2) in disease pathogenesis. Circ J 74(3):405-410

Jin M, Tong Q (2020) Rhabdomyolysis as Potential Late Complication Associated with COVID-19. Emerg Infect Dis 26(7)

Kim JE, Heo JH, Kim HO, Song SH, Park SS, Park TH, Ahn JY, Kim MK, Choi JP (2017) Neurological complications during treatment of Middle East respiratory syndrome. J Clin Neurol 13(3):227-233

Lau KK, Yu WC, Chu CM, Lau ST, Sheng B, Yuen KY (2004) Possible central nervous system infection by SARS coronavirus. Emerg Infect Dis 10(2):342-344

Li F (2016) Structure, function, and evolution of coronavirus spike proteins. Annu Rev Virol 3(1):237-261

Li Y, Li H, Fan R, Wen B, Zhang J, Cao X, Wang C, Song Z, Li S, Li X, Lv X, Qu X, Huang R, Liu W (2016) Coronavirus infections in the central nervous system and respiratory tract show distinct features in hospitalized children. Intervirology 59(3):163-169

Li YC, Bai WZ, Hashikawa T (2020a) The neuroinvasive potential of SARS-CoV2 may play a role in the respiratory failure of COVID-19 patients. J Med Virol. https://www.ncbi.nlm.nih.gov/pubmed/ 32104915

Li LQ et al (2020b) 2019 novel coronavirus patients' clinical characteristics, discharge rate, and fatality rate of meta-analysis. J Med Virol

Lu R, Zhao X, Li J, Niu P, Yang B, Wu H, Wang W, Song H, Huang B, Zhu N, Bi Y, Ma X, Zhan F, Wang L, Hu T, Zhou H, Hu Z, Zhou W, Zhao L, Chen J, Meng Y, Wang J, Lin Y, Yuan J, Xie Z, Ma J, Liu WJ, Wang D, Xu W, Holmes EC, Gao GF, Wu G, Chen W, Shi W, Tan W (2020) Genomic characterisation and epidemiology of 2019 novel coronavirus: implications for virus origins and receptor binding. Lancet 395(10224):565-574

Ludvigsson JF (2020) Systematic review of COVID-19 in children shows milder cases and a better prognosis than adults. Acta Paediatr

Madjid M et al (2020) Potential effects of coronaviruses on the cardiovascular system: a review. JAMA Cardiol

Mao L, Wang M, Chen S, He Q, Chang J, CHong C, et al. (2020) Neurological manifestations of hospitalized patients with COVID19 in Wuhan, CHina: a retrospective case series study. https://www. medrxiv.org/content/10.1101/2020.02.22.20026500v1.full.pdf

McIntosh K (1985) Coronaviruses. In: Fields BN et al (eds) Field's Virology. Raven Press, New York, pp 1323-1330

McIntosh K, Anderson LJ (2005) Coronaviruses, including severe acute respiratory distress syndrome (SARS)-associated coronavirus. In: Mandell GL, Bennett JE, Dolin R (eds) Mandell, Douglas, and Bennett's Principles and Practice of Infectious Diseases. Elsevier Churchill Livingston, Philadelphia, pp 1990-1997

Michael, B. and A. Easton. COVID-19 and encephalitis. 2020 [cited 2020 March 30]; Available from: www.encephalitis.info/blog/coronavirus

Netland J et al (2008) Severe acute respiratory syndrome coronavirus infection causes neuronal death in the absence of encephalitis in mice transgenic for human ACE2. J Virol 82(15):7264-7275 
Oke, J. and C. Heneghan. Oxford Covid-19 Evidence Service. 2020 [cited 2020 March 27]; Available from: https:/www.cebm.net/covid19/global-covid-19-case-fatality-rates/

Organization, W.H (2020) Middle East respiratory syndrome coronavirus (MERS-CoV). Emergencies [cited 2020; Available from: https:// www.who.int/emergencies/mers-cov/en/

Organization WH (2020) Coronavirus disease 2019 (COVID-19) Situation Report - 69. [cited 2020 March 29]; Available from: https://www.who.int/docs/default-source/coronaviruse/situationreports/20200329-sitrep-69-covid-19.pdf?sfvrsn=8d6620fa_2

Pan F, Ye T, Sun P, Gui S, Liang B, Li L, et al. (2020) Time Course of Lung Changes On Chest CT During Recovery From 2019 Novel Coronavirus (COVID-19) Pneumonia. Radiology 200370. https:// www.ncbi.nlm.nih.gov/pubmed $/ 32053470$

Poyuiadji N, Shahin G, Nouhjaim D, Stone M, Patel S, Griffith B (2020). COVID-19-associated acute hemorrhagic necrotizing encephalopathy: CT and MRI features. Radiology, from https://doi.org/10.1148/ radiol.2020201187

Seiden AM (2004) Postviral olfactory loss. Otolaryngol Clin N Am 37(6):1159-1166

Sharma K, Tengsupakul S, Sanchez O, Phaltas R, Maertens P (2019) Guillain-Barre syndrome with unilateral peripheral facial and bulbar palsy in a child: A case report. SAGE Open Med Case Rep 7: 2050313X19838750. https://www.ncbi.nlm.nih.gov/pubmed/ 30915222

Tan L, Wang Q, Zhang D, Ding J, Huang Q, Tang Y-Q, et al. (2020) Lymphopenia predicts disease severity of COVID-19: a descriptive and predictive study. Nature: Signal Transduction and Targeted Therapy [Internet]. https:/www.nature.com/articles/s41392-0200148-4.pdf.

Tang $\mathrm{N}$ et al (2020) Anticoagulant treatment is associated with decreased mortality in severe coronavirus disease 2019 patients with coagulopathy. J Thromb Haemost

Tang X, Wu C, Li X, Song Y, Yao X, Wu X, et al. (2020) On the origin and continuing evolution of SARS-CoV-2. Nat Sci Rev. https://doi. org/10.1093/nsr/nwaa036
Tian S, Hu N, Lou J, Chen K, Kang X, Xiang Z, et al. (2020) Characteristics of COVID-19 infection in Beijing. J Infect. https:// www.ncbi.nlm.nih.gov/pubmed/32112886

Tsai LK, Hsieh ST, Chang YC (2005) Neurological manifestations in severe acute respiratory syndrome. Acta Neurol Taiwanica 14(3): 113-119

Wang Y, Wang Y, Chen Y, Qin Q. (2020a) Unique epidemiological and clinical features of the emerging 2019 novel coronavirus pneumonia (COVID-19) implicate special control measures. J Med Virol. https://www.ncbi.nlm.nih.gov/pubmed/32134116

Wang D, Hu B, Hu C, Zhu F, Liu X, Zhang J, et al. (2020b) Clinical Characteristics of 138 Hospitalized Patients With 2019 Novel Coronavirus-Infected Pneumonia in Wuhan, China. JAMA. https://www.ncbi.nlm.nih.gov/pubmed/32031570

Wang D et al (2020c) Clinical characteristics of 138 hospitalized patients with 2019 novel coronavirus-infected pneumonia in Wuhan, China. JAMA

$\mathrm{Xu}$ XW et al (2020a) Clinical findings in a group of patients infected with the 2019 novel coronavirus (SARS-Cov-2) outside of Wuhan, China: retrospective case series. BMJ 368:m606

Xu H, Zhong L, Deng J, Peng J, Dan H, Zeng X et al (2020) High expression of ACE2 receptor of 2019-nCoV on the epithelial cells of oral mucosa. Int J Oral Sci. 12(1):8

Yang X et al (2020) Clinical course and outcomes of critically ill patients with SARS-CoV-2 pneumonia in Wuhan, China: a single-centered, retrospective, observational study. Lancet Respir Med

Yeh EA, Collins A, Cohen ME, Duffner PK, Faden H (2004) Detection of coronavirus in the central nervous system of a child with acute disseminated encephalomyelitis. Pediatrics 113(1 Pt 1):e73-e76

Zhao, K., et al., Acute myelitis after SARS-CoV-2 infection: a case report., m. preprint, Editor. 2020

Zhou F, Yu T, Du R, Fan G, Liu Y, Liu Z, et al. (2020) Clinical course and risk factors for mortality of adult inpatients with COVID-19 in Wuhan, China: a retrospective cohort study. Lancet

Publisher's note Springer Nature remains neutral with regard to jurisdictional claims in published maps and institutional affiliations. 